\title{
Establishing the Validity of the Severity Indicator Resource Utilization Scale in comparison to the Functional Independence Measure
}

\author{
Diana Velikonja* and Christine Brum
}

Acquired Brain Injury Program, Regional Rehabilitation Centre, Hamilton Health Sciences, Hamilton, Canada

\begin{abstract}
In the current study a resource utilization tool designed to assess the cognitive, behavioral and psychiatric consequences of brain injury, the SIRUS was compared to the FIM, which is commonly used resource utilization tool with established reliability and validity. Scores for brain injured patients were compared on both instruments as well as assessment of the psychometric properties of the SIRUS. The results demonstrated the SIRUS to be a reliable and valid tool for symptom assessment and evaluation in an inpatient $A B I$ (traumatic and non-traumatic) population. SIRUS scores discriminate between $A B I$ severity demonstrating non-traumatic brain injuries to be associated with poorer long term outcomes during inpatient rehabilitation. The findings would show the SIRUS to be designed for use in the $A B I$ population and is shown to be a quick and easily administered assessment tool with established reliability and validity that can accurately determine injury severity and the resource requirements of brain injured patients. The advantage of SIRUS over other resource utilization tools is that it uniquely accounts for the cognitive, behavioral and psychiatric issues related to brain injury which is not typically accounted for in other related instruments.
\end{abstract}

\section{Keywords: SIRUS; FIM; TBI; Non-traumatic TBI; ABI}

\section{Introduction}

Acquired brain injuries (ABI) are a significant cause of mortality and disability around the world. More than 10 million individuals worldwide sustain an ABI each year [1]. Approximately 70 to $90 \%$ of brain injuries are mild to moderate while 10 to $30 \%$ are severe [2]. Many of these individuals will experience problems with reintegration into home and community and some will require long-term assistance in activities of daily living (ADLs) and with instrumental activities of daily living (IADL) [3]. Intervention through appropriate rehabilitation resources can assist in decreasing length of hospital stay; reduce costs by minimizing the level of disability at discharge, enhancing functional independence, and increasing the number of patients who can be released into the home rather than a facility [4]. Many ABI survivors will suffer life-long disabilities that will significantly alter their lives [5]. As a result, substantial community based rehabilitation will be required in order to help them re-integrate back into the society. Recent changes in the provision of medical services, in particular rehabilitation services, for individuals with an $\mathrm{ABI}$ have highlighted the issue of limited resources available to these individuals post-hospitalization [5]. Therefore, in order to service this population optimally due to the chronicity of their impairments, it is important to accurately determine their resource needs to facilitate appropriate community management [5].

Measurement of functional activities and identifying disability are an important adjunct to medical treatments and are used as a communication tool between team members [6]. One of the difficulties with the assessment of functional activities is the lack of operational definitions for various tasks, how this information is condensed, and measurement accuracy. Therefore, it is argued that operational definitions need to be clearly written, provision for a description of what the observer should be looking for, and should contain precise procedures on how to assess the behavior [6]. Measurement quality is established through validity and reliability.

The Functional Independence Measure (FIM), is one of the most commonly used measurement tools in rehabilitation with established validity and reliability. The FIM is an 18-item, 7-level scale that rates the ability of an individual with disabilities to perform tasks such as, self-care, sphincter control, transfers, locomotion, communication, and social cognition independently [7]. Total scores are attained by summing scores across all 18 items. Scores can range from 18 , maximally dependent, to 126 , maximally independent. There are also two subscales, motor and cognitive, which can be scored by summing the 13 motor items (range 13-91) and the five cognitive items (range 5-35) [7]. FIM scores can be converted to percentage of care required by the patient, but reflects primarily their physical needs. As an outcome measure, FIM has become the most widely used indicator of disability severity, measure of burden of care as well as a resource utilization tool $[8,9]$. It has been described as providing a reasonable assessment of physical and psychosocial disability for brain injured individuals [10].

FIM has been shown to be effective and sensitive tools for assessing functional limitations [11]. It has demonstrated utility as an outcome measure for inpatient, community-based and comprehensive day rehabilitation programs, providing useful qualitative information. It also has demonstrated validity and reliability as an outcome measure [10]. For example, results from a meta-analysis of 11 studies estimating the reliability of the FIM indicated a median inter-rater reliability for the total FIM score of 0.95 and a median test re-test reliability of 0.95 [6].

In addition to measuring disability the capacity to capture clinically significant changes in the outcome measured is important (Houlden, Edwards, McNeil, \& Greenwood, 2006). This enables a valid measurement of case-mix pretreatment and subsequently the

*Corresponding author: Diana Velikonja, Acquired Brain Injury Program, Regiona Rehabilitation Centre, Hamilton Health Sciences, Hamilton, Ontario, Canada, Tel: 1-905-521-2100; E-mail: velikonja@hhsc.ca

Received September 27, 2013; Accepted October 17, 2013; Published October 27, 2013

Citation: Velikonja D, Brum C (2013) Establishing the Validity of the Severity Indicator Resource Utilization Scale in comparison to the Functional Independence Measure. J Neurol Neurophysiol 4: 169. doi:10.4172/2155-9562.1000169

Copyright: @ 2013 Velikonja D, et al. This is an open-access article distributed under the terms of the Creative Commons Attribution License, which permits unrestricted use, distribution, and reproduction in any medium, provided the original author and source are credited. 
effectiveness of treatment [12]. In terms of ABI/TBI, measurement must not only involve functional independence, physical impairments, but also a measure of the limitations caused by the cognitive and behavioral impairments which impact independence, especially in community re-entry [12]. Essentially, a measure must be able to establish the resources required for the individual to gain functional independence post-rehabilitation. Unfortunately, the number of tools available to measure resource utilization in ABI is scarce. Despite the fact that the FIM provides significant information regarding an individual's physical status it does not adequately capture the functional limitations imposed by cognitive and behavioral squeal [12].

The Severity Indicator Resource Utilization Scale (SIRUS) was created to encompass many of the positive aspects of FIM while also providing pertinent information about the level of assistance required and resources necessary for the functional limitations caused by cognitive, behavioral and psychiatric squeal. Thus SIRUS serves as a resource utilization tool more appropriate to the brain injured population. To help determine the validity of the SIRUS in the context of resource utilization management, it was compared to the FIM. Specifically, the FIM scores related to Communication (comprehension and expression) and Social Cognition (social interaction, problem solving, and memory) were compared to the SIRUS Behavior Status Domain and Cognitive Status Domain; while the Locomotion, Transfer, and Self-Care domains of the FIM were compared with the Physical Status Domain of SIRUS.

The objective of the current study is to first, compare SIRUS to FIM in terms of reliability and validity; and second, determine if the distributions of SIRUS items adequately distinguish patients admitted into rehabilitation. The third objective of this study is to determine the psychometric properties of SIRUS in order to establish whether or not they generalize to both traumatic and acquired brain injury patients. It is anticipated that SIRUS will not only maintain similar reliability and validity to that of the FIM but also provide greater insight into cognitive deficits.

\section{Methods}

\section{Participants}

Participants in the study were successively admitted inpatients to the Acquired Brain Injury Program (ABIP) at Hamilton Health Sciences (HHS) involving the Community Reintegration Unit (CRU) and the Centre for Behavioral Rehabilitation (CBR). The CRU is a transitional living unit where patients, who have typically sustained a moderate to severe ABI, and on average stay two to four months. The CBR is a behavioral rehabilitation unit with patients who have sustained primarily complex behavioral squeal related to their injury. Both units use a trans-disciplinary model of care in which regulated health professionals delegate aspects of assessment and treatment to non-regulated rehabilitation therapists trained to work with $\mathrm{ABI}$ patients. Data included all patients assessed by the Acquired Brain Program from January 2003 to August 2008. Eight hundred and sixty three patients were included in the present analyses. Five hundred and forty were male $(63.3 \%)$ and 313 were female $(36.7 \%)$. The sample consisted on 668 traumatic brain injuries $(78.3 \%)$ and 185 acquired brain injuries (21.7\%).

\section{Measures}

The Functional Independence Measure (FIM) is a measure of the assistance individuals require during the performance of activities of daily living (ADL), assessing self-care, sphincter management, transfers, locomotion, communication, and social cognition [13]. As an 18-item scale with a response category for each item ranging from 1 to 7, FIM scores evaluate patients complete dependence (score of 1) to complete independence (score of 7).

The SIRUS was created to assess the functional assessment to measure the level of assistance required as well as resources necessary. The SIRUS Behavior Status Domain and Cognitive Status Domain; and Locomotion, Transfer, and Self-Care (FIM) are compared with the Physical Status Domain.

\section{Procedure}

Data Analysis: Mean and Standard Deviation was determined for total SIRUS scores at admission. Scores were compared between each impairment category, Acquired Brain Injury Patients and Traumatic Brain Injury Patients. Descriptive statistics were also found for each of the following categories: Behaviors (A,B,C), Cognitive, Physical, Medical and Psychiatric. Factor analysis was completed for the Behavior Subscale and Cognitive Subscale of SIRUS across all categories. Correlations between each impairment category and subscale were also completed. In addition, average scores on each of the levels of SIRUS and the FIM were compared at Admission and Discharge.

\section{Results}

\section{Description of the sample}

Total SIRUS score distributions differ across traumatic and nontraumatic brain injury patients (i.e., cross-sectional discriminating ability) as demonstrated in Table 1 . This exhibits that the instrument can distinguish among different patient groups. Across the impairment categories, the non-traumatic group displayed high mean in the cognitive and medical status domain. Patients with non-traumatic injuries presented more severe symptoms that may disrupt daily activities (e.g. confusion, disorientation, impulsivity) and may have sporadic recurrence of identified medical issues that require monitoring.

\section{Factor analysis}

A maximum likelihood factor analysis with varimax rotation was conducted to explore the dimensional structure of the SIRUS scale. Results identified a two-factor solution (Table 2). The varimax rotation was used as it identifies items that are least correlated together. The two-factor solution revealed that Behavior A,B, and C items, as well as the psychiatric item were highly correlated together and could compose one item, while the cognitive, physical, and medical items were highly correlated and could compose another item. This two factor structure was validated when the sample was split by type of injury. For the remainder of this paper, factor 1 is termed behaviour subscale and factor 2 is termed cognitive subscale.

\begin{tabular}{|c|c|c|}
\hline Impairment Category & Non-Traumatic & Traumatic \\
\hline SIRUS total & $4.02(2.79)$ & $3.54(2.13)$ \\
\hline Behaviour A & $.23(.59)$ & $.15(.42)$ \\
\hline Behaviour B & $.27(.57)$ & $.30(.55)$ \\
\hline Behaviour C & $.15(.47)$ & $.09(.34)$ \\
\hline Cognitive & $1.24(.68)$ & $1.02(.60)$ \\
\hline Physical & $.68(.73)$ & $.75(.68)$ \\
\hline Medical & $1.07(.62)$ & $.89(.50)$ \\
\hline Psychiatric & $.39(.70)$ & \\
\hline
\end{tabular}

Table 1: Total SIRUS Score Mean and Standard Deviation at Admission. 


\begin{tabular}{|c|c|c|}
\hline & $\begin{array}{c}\text { Behaviour Subscale } \\
\text { (Factor 1) }\end{array}$ & $\begin{array}{c}\text { Cognitive Subscale } \\
\text { (Factor 2) }\end{array}$ \\
\hline $\begin{array}{c}\text { Total Sample: } \\
\text { Behaviour A }\end{array}$ & .655 & .195 \\
\hline Behaviour B & .592 & .119 \\
\hline Behaviour C & .549 & .013 \\
\hline Cognitive & .424 & .606 \\
\hline Physical & .058 & .562 \\
\hline Medical & .094 & .791 \\
\hline Psychiatric & .367 & .094 \\
\hline
\end{tabular}

Table 2: Factor Analysis Results.

\section{Descriptive Statistics and Correlations of Subscales for TBI} and ABI (Internal Consistency and Item Convergent Validity)

The descriptive statistics and the Cronbach's alphas for the behaviour and cognitive subscales for the two impairment categories as highlighted in Table 3. Non-traumatic ABI patients displayed higher means for both the behavior and cognitive subscale when compared to TBI patients. The Cronbach's alphas (internal consistency) between the two subscales for both impairment groups were relatively similar. The impairment categories further demonstrate high positive correlations $(\mathrm{r} \geq .75)$ to the total SIRUS score computed, demonstrating item convergent validity. TBI patients demonstrated lower correlations between the Total SIRUS and the behavior subscale when compared to the non-traumatic patients. While the non-traumatic patient's revealed lower correlations between the Total SIRUS and cognitive subscale when compared to the TBI patients.

\section{FIM and SIRUS}

The FIM is a commonly used measure that has been measured extensively by numerous researchers. As a reliable and valid measure, it is useful to compare the FIM and the SIRUS. Specifically, the FIM scores related to Communication (comprehension and expression) and Social Cognition (social interaction, problem solving, and memory) were compared to the SIRUS Behavior Status Domain and Cognitive Status Domain; and Locomotion, Transfer, and Self-Care (FIM) were compared with the Physical Status Domain.

Of the data that were released, 86 patients had complete data for all SIRUS and FIM items. In order to accurately compare the two measures, all scores were standardized. Table 4 presents the admission and discharge item averages. Participants demonstrated significant improvements from admission to discharge in five of the seven SIRUS scores: Behavior A $(\mathrm{t}(85)=4.85, \mathrm{p}<.001)$, Behavior B $(\mathrm{t}(85)=4.430$, $\mathrm{p}<.001)$, Cognitive $(\mathrm{t}(85)=11.54, \mathrm{p}<.001)$, Physical $(\mathrm{t}(85)=8.19, \mathrm{p}$ $<.001)$, and Medical $(\mathrm{t}(85)=12.35, \mathrm{p}<.001)$.

\section{Discussion}

The results of the current study demonstrate that the SIRUS has established psychometric properties which allow scores to distinguish impairment profiles consistently between brain injured patients. Thus scores could consistently and reliably differentiate the impairments displayed by $\mathrm{ABI}$ patients experiencing traumatic versus non-traumatic injuries with regard to injury severity and resource requirements. Interestingly, patients with non-traumatic injuries showed greater cognitive and medically based symptoms of greater severity than patients with traumatic injuries. This would indicate that patients that suffer non-traumatic brain injuries (i.e., disease, tumor, aneurysm, etc.) show poorer outcomes relative to those with traumatic injuries who appear to reveal a better trajectory of recovery as evaluated by the SIRUS. The second objective of the study was to review the psychometric properties of the SIRUS. Analysis of the instrument revealed that the instrument has a two dimensional structure. The first dimension was behavioral and psychiatric, indicating that symptoms in these two categories tend to be highly correlated in this population. Essentially, patients with high behavioral issues tend to show high psychiatric symptoms. The second dimension was cognitive, physical and medical, indicating the strong relationship between the severity of these symptoms in this population. The factor structure makes conceptual sense and is consistent with clinical outcomes in an inpatient rehabilitation setting. Thus in patients with higher cognitive symptoms also tend to have high physical and medical issues. ABI patients admitted to inpatient settings tend to have high multiple needs particularly in the acute stages of recovery. The findings showed psychometric values demonstrating good internal consistency and convergent validity of the SIRUS. Finally, SIRUS scores were able to accurately tract the trajectory of recovery for ABI inpatients, with results demonstrating consistent and highly correlated patterns of improvement over the course of treatment. Thus SIRUS scores were shown to be a valid and reliable measure of injury severity and resource utilization.

\begin{tabular}{|c|c|c|c|c|}
\hline & \multirow{2}{*}{ Mean } & \multirow{2}{*}{ SD } & \multicolumn{2}{|c|}{ Correlations } \\
\hline & & & Subscales & Total SIRUS \\
\hline \multicolumn{5}{|c|}{ Non-traumatic } \\
\hline Behaviour & .968 & 1.652 & $.329^{*}$ & $.819^{*}$ \\
\hline Cognitive & 3.104 & 1.623 & - & $.812^{*}$ \\
\hline \multicolumn{5}{|l|}{ Traumatic: } \\
\hline Behaviour & .932 & 1.277 & $.319^{*}$ & $.781^{*}$ \\
\hline Cognitive & .2 .678 & 1.472 & - & $.841^{*}$ \\
\hline
\end{tabular}

*. Correlation is significant at the 0.01 level (2-tailed).

Table 3: Subscale results across impairment categories.

\begin{tabular}{|c|c|c|}
\hline & Admission & Discharge \\
\hline & Mean (SD) & Mean (SD) \\
\hline \multicolumn{3}{|l|}{ SIRUS: } \\
\hline Behaviour A & $.69(1.03)$ & $.30(.65)$ \\
\hline Behaviour B & $.33(.68)$ & $.08(.27)$ \\
\hline Behaviour C & $.09(.33)$ & $.03(.18)$ \\
\hline Cognitive Domain & $2.77(.42)$ & $1.89(.81)$ \\
\hline Physical Domain & $2.15(1.06)$ & $1.44(1.18)$ \\
\hline Medical Domain & $2.09(.50)$ & $1.17(.65)$ \\
\hline Psychiatric Domain & $.14(.38)$ & $.20(.40)$ \\
\hline \multicolumn{3}{|l|}{ FIM: } \\
\hline \multicolumn{3}{|l|}{ Self- Care } \\
\hline Eating & $4.27(2.31)$ & $5.20(2.12)$ \\
\hline Grooming & $3.23(1.82)$ & $4.66(2.14)$ \\
\hline Bathing & $3.12(1.81)$ & $4.44(2.07)$ \\
\hline Dressing-Upper Body & $3.35(1.92)$ & $4.78(2.28)$ \\
\hline Dressing- Lower Body & $2.91(2.00)$ & $4.56(2.37)$ \\
\hline \multicolumn{3}{|l|}{ Locomotion } \\
\hline Walk/Wheelchair & $3.61(2.44)$ & $5.08(2.22)$ \\
\hline Stairs & $2.91(2.29)$ & $4.28(2.47)$ \\
\hline \multicolumn{3}{|l|}{ Communication } \\
\hline Comprehension & $3.72(1.48)$ & $4.74(1.36)$ \\
\hline Expression & $3.43(1.55)$ & $4.44(1.54)$ \\
\hline \multicolumn{3}{|l|}{ Social Cognition } \\
\hline Social Interaction & $3.47(1.78)$ & $4.50(1.77)$ \\
\hline Problem Solving & $2.05(1.23)$ & $3.67(1.75)$ \\
\hline Memory & $2.36(1.41)$ & $3.93(1.90)$ \\
\hline
\end{tabular}

Note. Sphincter Control and Transfer Data were not used in comparisons.

Table 4: Admission and Discharge Standardized Average Scores for the FIM and SIRUS. 
Citation: Velikonja D, Brum C (2013) Establishing the Validity of the Severity Indicator Resource Utilization Scale in comparison to the Functional Independence Measure. J Neurol Neurophysiol 4: 169. doi:10.4172/2155-9562.1000169

Overall SIRUS has been shown to be a valid and reliable tool in symptom assessment and evaluation over time in an inpatient ABI population. This tool can discriminate between more severe presentations of $\mathrm{ABI}$ which were shown to be distinguished along the traumatic versus non-traumatic dimension, revealing poorer long term outcomes for the non-traumatic group. This tool was designed for use in the ABI population and is shown to be a quick and easily administered assessment tool with established reliability and validity that can accurately determine injury severity and the resource requirements of brain injured patients. The advantage of SIRUS over other resource utilization tools is that it uniquely accounts for the cognitive, behavioral and psychiatric issues related to brain injury which is not typically accounted for in other related instruments.

\section{References}

1. Tate RL (2004) Assessing support needs for people with traumatic brain injury: the Care and Needs Scale (CANS). Brain Inj 18: 445-460.

2. Borg J, Holm L, Peloso PM, Cassidy JD, Carroll LJ, et al. (2002) Non-surgical intervention and cost for mild traumatic brain injury: results of the WHO Collaborating Centre Task Force for Mild Traumatic Brain Injury. Journal of Rehabilitation Medicine 43: 76-83.

3. Bottari C, Dassa C, Rainville C, Dutil E (2009) The factorial validity and internal consistency of the Instrumental Activities of Daily Living Profile in individuals with traumatic brain injury. Neuropsychological Rehabilitation 19: 177-207.

4. Lequerica AH, Rapport LJ, Whitman RD, Millis SR, Vangel SJ, et al. (2006) Psychometric properties of the Rehabilitation Therapy Engagement Scale when used among individuals with acquired brain injury. Rehabilitation Psychology 51: 331-337.
5. Labi ML, Brentiens M, Coad ML, Flynn WJ, Zielezny M (2003) Development of a longitudinal study of complications and functional outcomes after traumatic brain injury. Brain Inj 17: 265-278.

6. Cullen NK, Park YG, Bayley MT (2008) Functional recovery following traumatic vs non-traumatic brain injury: a case-controlled study. Brain Inj 22: 1013-1020.

7. Unsworth CA (2001) Selection for rehabilitation: acute care discharge patterns for stroke and orthopaedic patients. Int J Rehabil Res 24: 103-114.

8. Dallmeijer AJ, Dekker J, Roorda LD, Knol DL, van Baalen B, et al. (2005) Differential item functioning of the Functional Independence Measure in higher performing neurological patients. J Rehabil Med 37: 346-352.

9. Gosman-Hedström G, Blomstrand C (2004) Evaluation of a 5-level functiona independence measure in a longitudinal study of elderly stroke survivors. Disabil Rehabil 26: 410-418.

10. Law J, Fielding B, Jackson D, Turner-Stokes L (2009) The UK FIM+FAM Extended Activities of Daily Living module: evaluation of scoring accuracy and reliability. Disabil Rehabil 31: 825-830.

11. Wilson FC, Wheatley-Smith L, Downes C (2009) Analysis of intensive outpatien neuro-rehabilitation outcomes using FIM+FAM(UK). NeuroRehabilitation 24 377-382.

12. Houlden H, Edwards M, McNeil J, Greenwood R (2006) Use of the Barthel Index and the Functional Independence Measure during early inpatient rehabilitation after single incident brain injury. Clinical Rehabilitation 20: 153-159.

13. Brock KA, Goldie PA, Greenwood KM (2002) Evaluating the effectiveness of stroke rehabilitation: choosing a discriminative measure. Arch Phys Med Rehabil 83: 92-99. 